\title{
Surface sensitive x-ray spectroscopy (TREXS) for nanoscale surface study and multi-modal development
}

\author{
H. Abe $e^{1,2,3}$, Y. niwa ${ }^{1}$, M. Kimura ${ }^{1,2}$ \\ ${ }^{1}$ Institute of Materials Structure Science, High Energy Accelerator Research Organization (KEK), 1-1 Oho, Tsukuba, Japan, \\ ${ }^{2}$ SOKENDAI (The Graduate University for Advanced Studies), 1-1 Oho, Tsukuba, Japan, \\ ${ }^{3}$ Graduate School of Science and Engineering, Ibaraki University, 2-1-1, Bunkyo, Mito, Ibaraki, Japan \\ hitoshi.abe@kek.jp
}

X-ray absorption fine structure (XAFS) spectroscopy is one of the most widely used synchrotron radiation based methods to study local structures and electronic states of elements. Chemical reactions have been observed by in situ XAFS methods. However, XAFS is fundamentally bulk sensitive, and is difficult to apply to study surface phenomena or reactions.

We have developed a surface sensitive $\mathrm{x}$-ray spectroscopy, which is named Total REflection X-ray Spectroscopy (TREXS), to study surfaces in nanometer scale. Reflection spectra are recorded in total reflection conditions, and the surface sensitivity of about 2-3 nm is realized. In brief, TREXS enables us to obtain two kinds of information, which essentially correspond to usual XANES and EXAFS. Near edge regions of total reflection spectra are analyzed to discuss electronic structures and chemical states, and surface reactions can be monitored by tracking the changes. In addition, total reflection spectra are transformed to XAFS spectra through Kramers-Kronig relations, and regular EXAFS analysis methods can be applied.

A reduction reaction of surface $\mathrm{NiO}$ layer to Ni metal was observed with the high surface sensitivity of $\sim 2-3 \mathrm{~nm}$ as shown in Figure 1 [1,2]. A surface oxidized Ni film was placed in a home-made in situ TREXS cell. The grazing incident angle of x-ray was adjusted to be $\sim 2.5 \mathrm{mrad}$, which is well below the critical angle of total reflection. The surface reduction reaction was observed under the condition of mixture gas of $\mathrm{H}_{2} 30 \mathrm{sccm}$ and $\mathrm{N}_{2} 90 \mathrm{sccm}$ at $540 \mathrm{~K}$. In addition, a reduction reaction of surface Co oxide layer to Co metal was reported [3].

In this contribution, we will present surface chemical reactions studied by in situ TREXS, development of multi-modal surface research equipment by combining TREXS with IRRAS (Infrared Reflection Absorption Spectroscopy), and an ongoing plan to involve also scattering techniques in the TREXS experimental equipment. This will lead to develop an experimental setup to study surfaces in nanometer scale by spectroscopy (TREXS) and scattering including diffraction at the same time under reaction conditions.

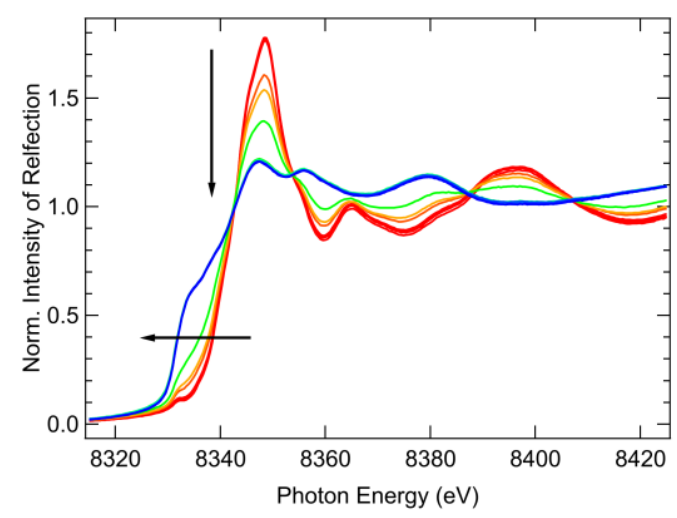

Figure 1. Spectra recorded during in situ TREXS measurement of a reduction reaction of $\mathrm{NiO}$ to $\mathrm{Ni}$ metal.

[1] Abe, H., Nakayama, T., Niwa, Y., Nitani, H., Kondoh, H. \& Nomura, M. (2016). Jpn. J. Appl. Phys. 55, 062401.

[2] Abe, H., Niwa, Y., Takeichi, Y. \& Kimura, M. (2019) Chem. Rec. 19, 1457.

[3] Abe, H., Niwa, Y., \& Kimura, M. (2020). Phys. Chem. Chem. Phys. 22, 24974.

Keywords: XAFS; Surface; Total Reflection; in situ; nanometer scale

This work was performed under the approval of PF-PAC No. 2011G116, 2013G546, 2015G551, $2017 G 686$ and 2019G676. The authors are grateful for the financial support from JSPS KAKENHI Grant Number 24710102 and $16 H 05992$.

Acta Cryst. (2021), A77, C1061 